\title{
Nonquenched rotators ease flocking and memorise it
}

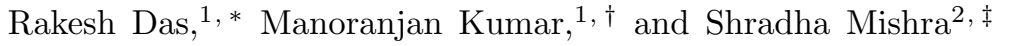 \\ ${ }^{1} S$ N Bose National Centre for Basic Sciences, Block JD, Sector III, Salt Lake, Kolkata 700106, India \\ ${ }^{2}$ Department of Physics, Indian Institute of Technology (BHU), Varanasi 221005, India
}

We introduce a minimal model for a two-dimensional polar flock with nonquenched rotators, and show that the rotators make the usual macroscopic long-range order of the flock more robust than the clean system. The rotators memorise the flock-information which helps in establishing the robustness. Moreover, the memory of the rotators assists in probing the moving flock. We also formulate a hydrodynamic framework for the microscopic model that makes our study comprehensive. Using linearised hydrodynamics, it is shown that the presence of such nonquenched heterogeneities increases the sound speeds of the flock. The enhanced sound speeds lead to faster convection of information and consequently the robust ordering in the system. We argue that similar nonquenched heterogeneities may be useful in monitoring and controlling large crowds.

\section{INTRODUCTION}

Collective behaviour of large-scale systems like crowd of pilgrims [1 5 and flock of birds [6] spanning a few kilometers, or micron-scale population of bacteria [7, 8, display many common features like phase segregation [9] 11], large density fluctuations [12, etc. These features stem from the self-propulsion nature of the individual constituents exhibiting systematic movement at the cost of their internal energy [13 17. A comprehensive understanding of these systems have immense utility [18, in various aspects of society, viz., safety measures of human crowds [1 5], cell biology [19, 20] and drug delivery employing microrobotics [21, 22]. Extensive theoretical and experimental studies have indeed developed a primary insight of the underlying physics in the clean systems [13 17, 23, 27. However, heterogeneity is inevitable in natural systems [28, e.g., bacteria moving on complex substrates [29, 30], human traffic with varieties of obstacles [31, 32]. Recently, various heterogeneous systems are studied, and it is shown that usually the collective movement of the flock gets hampered by the heterogeneities 33 39. Surprisingly, specific type of external agents can also help in flock formation or crowd control 4043. However, a generic framework for the heterogeneous self-propelled system is lacking in the literature which may help in understanding varieties of flocking systems.

Study of the clean self-propelled systems using minimal rule-based microscopic models has been successful in predicting and explaining many features therein 22,44 . Inspired by this, in this article, we propose a minimal model for a heterogeneous self-propelled system. The heterogeneity agents are modeled as inertial rotators which try to retain their orientations and affect the neighbouring flock. However, the flock also gives feedback of its orientational information to the rotators and changes their orientations. Our numerical study reveals that an in-

\footnotetext{
* rakeshd68@yahoo.com

$\dagger$ manoranjan.kumar@bose.res.in

$\ddagger$ smishra.phy@itbhu.ac.in
}

terplay of the feedback mechanism and the inertia of the rotators build a correlated flock which is more robust to fluctuations than the clean system. Moreover, these rotators memorise the flock orientation that offers a novel mechanism for probing the flock statistics. We also provide a hydrodynamic description of this minimal model. The linearised calculations show that these heterogeneities effectively increase the convection speed that establishes the robust ordering in the system.

\section{MODEL}

We consider a collection of $N_{s}$ polar self-propelled particles (SPPs) on a two-dimensional (2D) substrate. These particles are characterised by their instantaneous positions $\boldsymbol{r}_{j}(t)$ and orientations $\theta_{j}(t)$. Each SPP tries to orient parallel to its neighbours, although it makes errors. The particles move along their updated orientations with a constant speed $v_{s}$. The substrate is also populated with $N_{r}$ randomly-placed nonquenched rotators (NRs). The NRs remain stationary and have their own orientations $\phi_{j}(t)$. The $j$-th NR influences its neighbouring SPPs and tries to reorient those SPPs along $\phi_{j}$. In turn, the flock of the SPPs also tries to reorient the NR along the mean flock-orientation. However, the effect of the SPPs on a NR is suppressed by its inertia, as the NR tries to retain its earlier orientation. Therefore, the model is described by the following update rules:

$$
\begin{aligned}
\theta_{j}(t+1) & =\arg \left[\sum_{k \in R} e^{i \theta_{k}(t)}+\mu \sum_{k \in R} e^{i \phi_{k}(t)}\right]+\eta_{\theta} \psi_{\theta}, \\
\boldsymbol{r}_{j}(t+1) & =\boldsymbol{r}_{j}(t)+\boldsymbol{v}_{j}(t+1), \\
\phi_{j}(t+1) & =\arg \left[e^{i \phi_{j}(t)}+\alpha \sum_{k \in R} e^{i \theta_{k}(t)}\right]
\end{aligned}
$$

where $\arg [\boldsymbol{z}]$ represents the argument $\Theta$ of $\boldsymbol{z}=\mathcal{R} e^{i \Theta}$. The self-propulsion velocity $\boldsymbol{v}=v_{s}(\cos \theta(t), \sin \theta(t)), R$ represents the interaction radius, and summations are considered over all neighbours within $R$. The mutual interactions among the SPPs and the NRs are tuned by 
the strengths $\mu$ and $\alpha$. We choose these two parameters mutually independent due to the lack of momentum conservation in the dry active systems [13. Errors in the process of orientation update of the SPPs are incorporated by an uniform additive noise $\psi_{\theta}$ in the range $[-\pi, \pi]$ with zero mean and white correlations. $\eta_{\theta} \in[0,1]$ represents the noise strength. The rotators are called nonquenched as their orientations are changed by feedback of the flockorientations.

The effect of the neighbouring particles on a tagged SPP (NR) is schematically presented in Fig. 1. For an arbitrary configuration shown in the top panel of Fig. 1 1a (respectively, b), the effective interactions are depicted in the bottom panel after properly tuned by $\mu$ (respectively, $\alpha$ ). Due to the interactions with the neighbours, the orientation of the tagged particle would be updated towards the resultant direction, as represented by the black arrow. We evidence that due to the presence of the NRs, a robust coordination is developed among the SPPs. The corresponding mechanism is depicted in the schematic Fig. 10, which we discuss in Sec. III A.

The above model is similar to the celebrated Vicsek model 23 in the absence of the NRs, and it describes a clean flock where a true long-range order (LRO) exists in 2D [24, 25. Also note that the rotators become quenched for $\alpha=0$, and therefore, no long-range order but a quasilong-range order (QLRO) may survive in the system 3739.

\section{RESULTS}

\section{A. Robust long-range order}

We simulate the update Eqs. (1)-(3) numerically in a $L \times L(L \in[100,500])$ substrate with periodic boundaries. $N_{s}$ SPPs (density $c_{s}=N_{s} / L^{2}=1$ ) and $N_{r}$ NRs (density $c_{r}=N_{r} / L^{2}$ ) with random orientations are distributed on the substrate. As we are interested in the physical limit where heterogeneities are small in numbers as compared to the SPPs, we restraint ourselves to $c_{r} \in[0,0.045]$. Further, we consider $v_{s}=1$ and $R=1$, and execute a code exploiting OpenMP application program interface. We note that depending on the system size, $3 \times 10^{5}$ to $16 \times$ $10^{5}$ iterations are sufficient to attain steady states, and calculate the relevant quantities by averaging over next $5 \times 10^{5}$ to $14 \times 10^{5}$ iterations. Upto 30 realisations are used for better statistics. The order parameter of the SPPs $V_{s}=\left\langle V_{s}(t)\right\rangle_{t}=\left\langle\frac{1}{N_{s}}\left|\sum_{j=1}^{N_{s}} e^{i \theta_{j}(t)}\right|\right\rangle_{t}$ varies from zero to unity for a disordered to an ordered state, respectively.

The clean system $\left(c_{r}=0\right)$ shows a monotonic orderdisorder transition with increasing $\eta_{\theta}$ (Fig. 2a). However, in the presence of the quenched rotators $(\alpha=0)$, the system achieves optimal ordering at a nonzero $\eta_{\theta}$. This optimality feature emerges as the quenched rotators disturb transfer of informations among the SPPs, and the system needs a certain noise to circumvent that hin- (a)
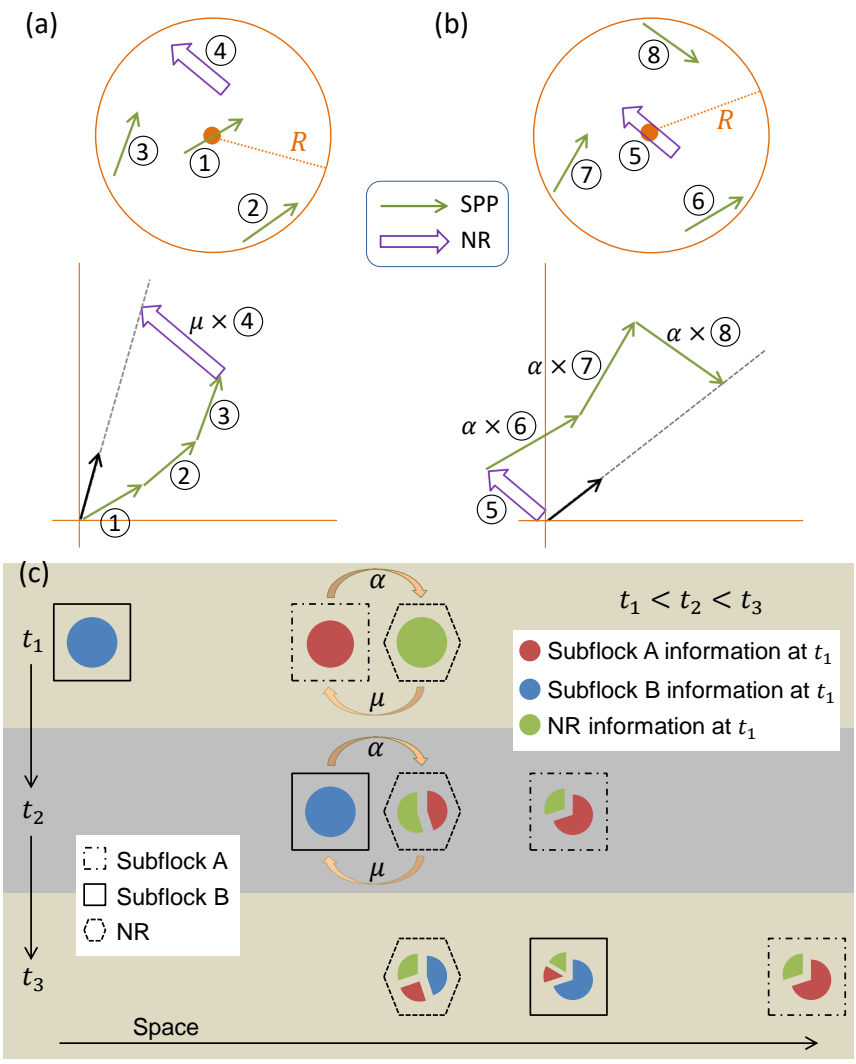

Figure 1. Schematic of interaction among flocks and NR. (a) Top - A test SPP (' 1 ') interacts with its neighbours ('2, $\left.3,4^{\prime}\right)$ within a distance $R$. Bottom - Vectorial representation of the orientation update rule of the SPP (' 1 '). The SPP-NR (' 1 '-'4') interaction is tuned by $\mu$. The dashed line shows the resultant orientation and the black arrow on it represents the corresponding unit vector. (b) Similar presentation for the orientation update of a test NR (' 5 '). The NR-SPP interactions are tuned by $\alpha$. (c) Two subflocks (A - broken square, B - solid square) of SPPs and a NR (broken hexagon) are considered in an arbitrary $2 \mathrm{D}$ space at time $t_{1}$. The colours inside the geometries indicate informations therein. All the informations are called pure at $t_{1}$. As A and NR are nearby at $t_{1}$, they interact mutually through $\mu$ - and $\alpha$-terms, and meanwhile $\mathrm{B}$ remains reclusive. During interactions, A and NR exchange informations as per the values of $\mu$ and $\alpha$. A passes by the NR outside its interaction range by $t_{2}$. By that time, B comes close to NR and interact mutually. Consequently, NR receives information of $\mathrm{B}$, and transfer its current information (which also contains information of A) to B, as depicted for a late time $t_{3}$. Other than direct interactions among $\mathrm{A}$ and $\mathrm{B}$, this extra means of information transfer through the inertia of NR establishes ordering robust than the clean system. Also, NR acts as probe to the flocks as it memorises informations of the flocks.

drance [34, 37. As the optimal $\eta_{\theta}$ increases with $\mu$, we set $\mu=200$ such that the optimal $\eta_{\theta}$ attain moderate values for the studied range of $c_{r}$. In contrast to the quenched model, the system shows a monotonic transition for $\alpha>0$ in the presence of the NRs. Similar to the clean case [23, 26, we note a homogeneous ordered state of SPPs in 
(a)
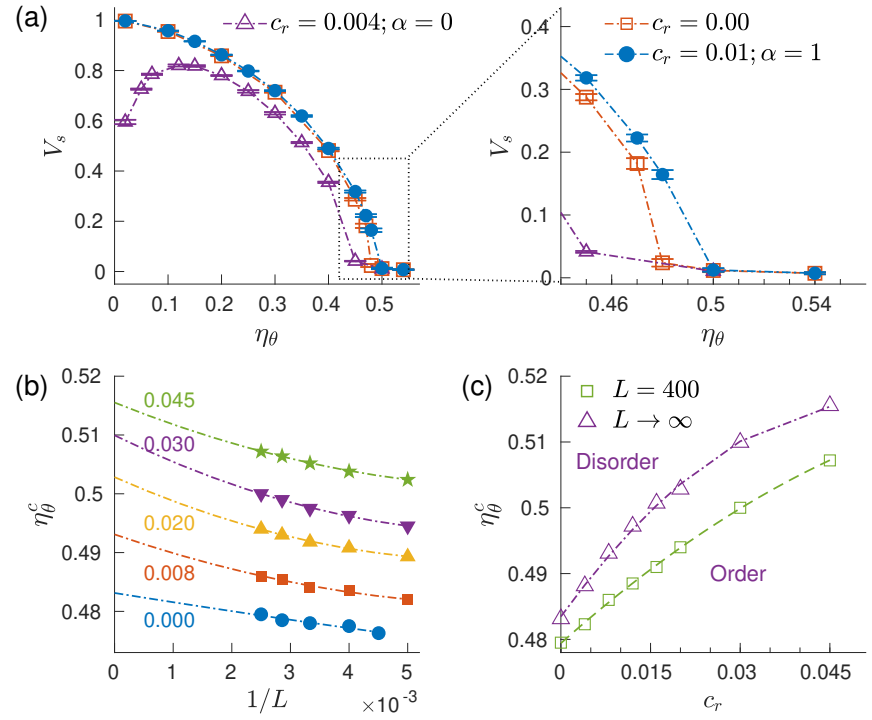

(c)

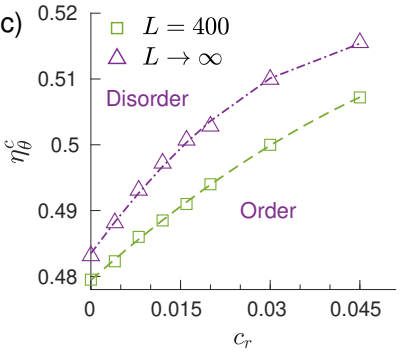

Figure 2. Robust ordering due to NRs. The data are shown for $\mu=200$. (a) The SPPs show monotonic order-disorder transition with $\eta_{\theta}$ in the presence of the NRs $\left(c_{r}=0.01, \alpha=\right.$ $1)$, similar to the clean system $\left(c_{r}=0\right)$. Unlike that, the quenched model $\left(c_{r}=0.004, \alpha=0\right)$ shows nonmonotonic transition. The curves are zoomed near the transition on the right panel of (a). (b) Critical noise $\eta_{\theta}^{c}$ increases with $L$. The numerics on the left margin indicate $c_{r}$, and the dashed lines show respective quadratic fits. The thermodynamiclimit value $\eta_{\theta}^{c}(L \rightarrow \infty)$ of the critical noise is obtained from the extrapolation. (c) $\eta_{\theta}^{c}(L)$ increases quadratically with density $c_{r}$. The broken lines show respective fits. Order and disorder states of the SPPs are indicated for the thermodynamic limit.

the presence of the NRs for noises much smaller than its critical value for the order-disorder transition. We also note that banded configuration emerges near the critical noise where a highly ordered dense cluster of SPPs travels over disordered sparse background [26, 45]. Surprisingly, the system with the NRs survives upto $\eta_{\theta}$ higher than its clean counterpart, as the zoomed version of Fig. $2 \mathrm{a}$ shows. This necessarily implies that the flock is more robust to the external fluctuations $\left(\eta_{\theta}\right)$ as compared to the clean system. To ensure this emerged robustness, we calculate the critical noise $\eta_{\theta}^{c}$ of the order-disorder transition and compare it for several $c_{r}$. The Binder cumulant [26, 45, 46] $U(L)=1-\left\langle V_{s}^{4}(t, L)\right\rangle_{t} / 3\left\langle V_{s}^{2}(t, L)\right\rangle_{t}$ shows a dip to negative values near the transition (Appendix A). We define $\eta_{\theta}^{c}$ as the noise corresponding the $\min (U(L))$. We check that reduced critical noise $\epsilon=1-\eta_{\theta}^{c} / \eta_{\theta}^{c \mathcal{L}}$ scales as $L^{-\zeta}$, where $\eta_{\theta}^{c \mathcal{L}}$ represents critical noise obtained for $L=400$ (data not shown). We note that the finite size scaling (FSS) exponent $\zeta=2$ for the clean system, as reported in Ref. [26], and the exponent increases for $c_{r}>0$. Though it is interesting to check the variation in $\zeta$ with $c_{r}$, a rigorous FSS study of the present Vicsek-like model with angular noise [26] is numerically expensive and beyond our current objective. Rather, we note that $\eta_{\theta}^{c}$ increases quadratically with decreasing $1 / L$ (Fig. $2 \mathrm{~b}$ ).
So, the thermodynamic limit values $\eta_{\theta}^{c}(L \rightarrow \infty)$ of the critical noises are obtained from extrapolations of these curves to the $L \rightarrow \infty$ limit. Interestingly, $\eta_{\theta}^{c}(L \rightarrow \infty)$ increases quadratically with $c_{r}$ (Fig. 2k), and therefore, the NRs indeed offer a mechanism for a flock to be more robust than the clean system. We also plot the $\eta_{\theta}^{c}-c_{r}$ curve for $L=400$ for comparison, and note similar behaviour as explained for the thermodynamic limit.

The order parameter of the clean system does not depend on the system size (Fig. 3a) which is a manifestation of the LRO therein [23 25, 37]. We also note that in the presence of the quenched rotators, $V_{s}$ decreases algebraically with $N_{s}$, implying a QLRO state in the system [37 39]. However, $V_{s}$ does not change with $N_{s}$ in the presence of the NRs. Moreover, the order parameter in the presence of the NRs is larger than clean system. Therefore, similar to the clean system, a LRO exists in the presence of the NRs, and that state is more robust than the clean system. The nature of the ordered state is further confirmed by calculating a normalized distribution $P(\theta)$ of the SPP-orientations for various system sizes 37. This distribution is a measure of the orientation fluctuations among the SPPs, and it does not vary with the system size in the presence of the NRs (Fig. 3b). However, $P(\theta)$ widens with system size in the quenched model as there exists a QLRO only [37.

The mechanism that makes the LRO state more robust than the clean system can be understood from the schematic Fig. 1p drawn on an arbitrary 2D space. Two subflocks A and B, and a NR have their pure orientational informations at time $t_{1}$, as represented by the colours red, blue and green, respectively. While B is away from the NR, A and NR interact mutually through the $\mu$ - and $\alpha$ terms and exchange informations. Therefore, at a later time $t_{2}, \mathrm{~A}$ and NR contain both the pure informations (red and green) corresponding to time $t_{1}$. The proportion of this exchange is determined by the parameters

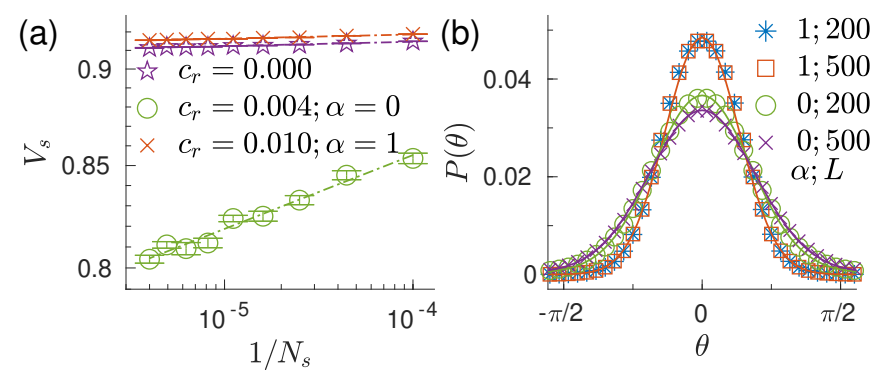

Figure 3. LRO in the presence of NRs. The data are shown for $\mu=200$ and $\eta_{\theta}=0.15$. (a) The SPP-order parameter does not vary with system size for the clean system $\left(c_{r}=0\right)$ or the system with the NRs $\left(c_{r}=0.01, \alpha=1\right)$. However, in the presence of the quenched rotators $\left(c_{r}=0.004, \alpha=0\right)$, the order parameter decreases algebraically. The lines show respective algebraic fits. (b) The orientation distribution of the SPPs do not depend on the system size in the presence of the NRs, whereas the distribution broadens with $L$ for the quenched model. 
$\mu$ and $\alpha$. However, by that time A moves beyond the interaction range of the $\mathrm{NR}$, and $\mathrm{B}$ comes close to the same NR. Due to a similar kind of interaction through $\mu$ and $\alpha$, the NR now receives the pure information of $\mathrm{B}$ (blue). In turn, $\mathrm{B}$ receives the pure information of $\mathrm{A}$ (red) even without any direct interaction, as shown in the schematic for time $t_{3}$. Therefore, in the duration of $t_{1}$ to $t_{3}$, the NR has received feedback from the subflock $\mathrm{A}$ and later it delivered that information to $\mathrm{B}$ through its inertia. In addition to the usual convection of the SPPs [23, 25], this mechanism provides an extra means of information transfer among the particles that induces robustness in the system. Also note that the NR memorises the informations of the passing by flocks, which is beneficiary for probing the flocks through these external agents, as we discuss next.

\section{B. NRs probe the flock}

We investigate the orientation autocorrelation of the NRs, defined as $C_{\phi}(t)=\left\langle\cos \left[\phi_{j}\left(t+t_{0}\right)-\phi_{j}\left(t_{0}\right)\right]\right\rangle_{j, t_{0}}$. Here $\langle\cdot\rangle$ symbolizes averaging over all the NRs and many steady-state reference times $t_{0}$. Starting from an orientation $\phi_{j}\left(t_{0}\right), j$-th NR changes its orientation due to the feedback from the flocks. Therefore, $C_{\phi}$ shows an earlytime decay, and beyond that it saturates to the square of the order parameter $V_{r}$ of the NRs. $V_{r}$ is defined similar to $V_{s}$. The early-time decay in $C_{\phi}$ indicates the timescale upto which a NR remembers its earlier orientation. This timescale is necessarily dictated by $\alpha$, as Eq. (3) suggests. $V_{r}$ depends on $\eta_{\theta}$ through the feedback from the flock, and also on $\alpha$. However, $V_{s}$ does not change significantly with $\alpha$, provided $\alpha>0$. Therefore, we stress that the flock-phenomenology described here and its implications hold for any finite value of $\alpha$. The results presented in this article are obtained for $\alpha=1$.

We note that $C_{\phi}$ shows periodic modulations over its saturated value (Fig. 4 a). This is more prominent near the order-disorder transition where band emerges in the system (Fig. 4b-g) 26, 47, 48, The modulations in $C_{\phi}$ offer a novel mechanism of probing the statistics of the flock. Let us consider a banded-state configuration at a reference time $t_{0}$ when all the NRs have their initial orientations $\phi_{j}\left(t_{0}\right)$ (Fig. $4 \mathrm{p}$ ). The NRs inside the band have a spatially correlated distribution of orientations due to the feedback from the correlated SPPs. However, the NRs outside the band have random $\phi_{j}\left(t_{0}\right)$. Though $\phi_{j}$ changes as the band moves forward, the same NRs that are still inside the band (Fig. 4k) remains correlated to their initial orientations. The rest of the NRs yield a zero contribution to $C_{\phi}$ as they have been averaged over random terms. Therefore, we obtain a finite $C_{\phi}$ in aggregate. The band moves further in the course of time (Fig. $4 \mathrm{~d}$-e), and there exists no finite contribution to $C_{\phi}$ until the band-front reaches (due to periodic boundary condition) the region of the band-tail of time $t_{0}$ (Fig. 4 e). $C_{\phi}$ again becomes finite with time (Fig. 4f), and it is the

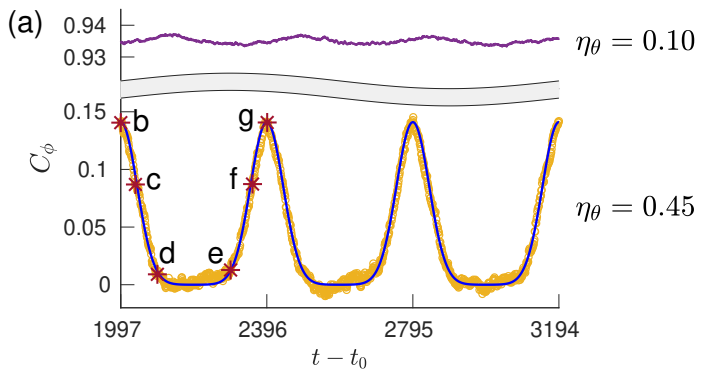

(b)

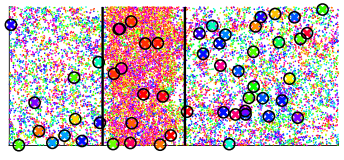

(c)

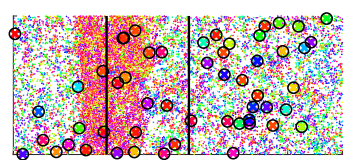

(d)

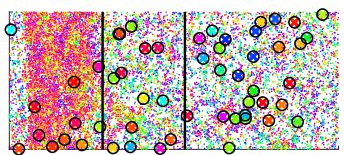

(e)

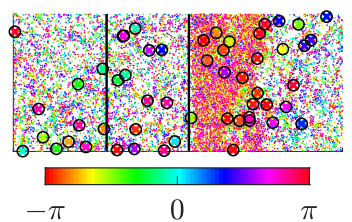

(f)

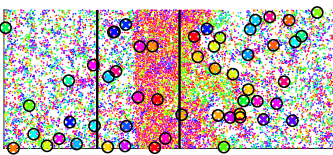

(g)

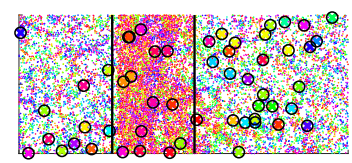

(h)

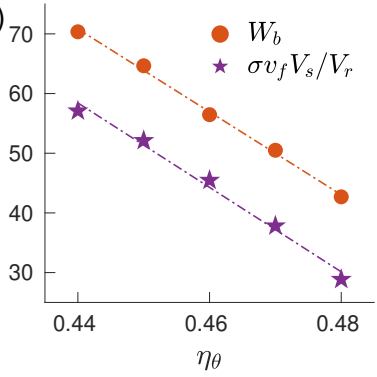

Figure 4. Flock information is stored in the NRs. The data are shown for $\mu=200$. (a) Autocorrelation function $C_{\phi}$ of the NRs $\left(c_{r}=0.01, \alpha=1, L=300\right)$ are shown at the late time, $t_{0}$ being the reference steady-state time. $C_{\phi}$ shows modulations over its saturated value $V_{r}^{2}$, and these modulations are prominent near the critical point $\left(\eta_{\theta}=0.45\right)$ where band emerges. The solid line shows Gaussian fit to these modulations, and the labels correspond to the snapshots shown in (b-g). The snapshots are shown for a zoomed substrate of dimension $300 \times 100$ for clarity. The black circles indicate the NRs (only few shown), and the colours represent orientations of the SPPs and the NRs. Separation in the black vertical lines in (b) gives an estimate of the bandwidth $W_{b} . C_{\phi}$ assumes a finite value only if the current position of the band overlaps to that in (b). (h) $W_{b}$ and $\sigma v_{f} V_{s} / V_{r}$ decreases with $\eta_{\theta}$ with the same slope. Here the flock speed $v_{f}=L / \tau$ where $\tau$ is the peak-to-peak separation of $C_{\phi}$ modulations.

maximum when the band reaches the same position as $t_{0}$ (Fig. 4s). Following this, the same dynamics continues. Therefore, the peak-to-peak separation $\tau$ in $C_{\phi}$ modulations (Fig. $4 \mathrm{~b}$ ) is the time required by the flock (density wave) to traverse the system once (Supplementary Movie [49]). We obtain the flock (band) speed $v_{f}$ from the ratio of $L$ and $\tau$. The modulations in $C_{\phi}$ shows good fit with Gaussian curves (Fig. 4a). Standard deviation $\sigma$ of the Gaussian fit multiplied by $v_{f}$ shows a linear decay with $\eta_{\theta}$ (Fig. $4 \mathrm{~h}$ ). Note that $\sigma v_{f}$ is a NR-property that should give a measure of the width of the band, as the finite contributions to $C_{\phi}$ are due to the band. Next, 
we verify that $\sigma v_{f}$ indeed bears the informations of the band.

We divide the whole substrate into $L$ strips of unit width parallel to the band. The ratio of the number of SPPs inside a strip to $L$ gives the local density $c_{s}^{l o c}$ therein. The lateral dimension for which $c_{s}^{l o c}>c_{s}$ is defined as the bandwidth, which is similar to the length between the black vertical lines in Fig. $4 \mathrm{~b}$. We calculate the mean bandwidth $W_{b}$ averaged over many snapshots and see that $W_{b}$ decreases linearly with $\eta_{\theta}$ with the same slope as $\sigma v_{f} V_{s} / V_{r}$ versus $\eta_{\theta}$ curve (Fig. 4h). Therefore, $\sigma v_{f}$ which is a NR-property indeed bears the flock information, and the NRs act as footprints of the passed-by flock. Note that $W_{b}$ is calculated considering clustering of the SPPs, whereas $\sigma v_{f}$ contains information of the ordering in the system. Therefore, we need the multiplication factor - relative strength of order of the SPPs and the NRs $\left(V_{s} / V_{r}\right)$ to compare a NR-property $\sigma v_{f}$ with a flock-property $W_{b}$.

Note that the bands may arise in any direction. However, we present the case of the lateral bands only, and emphasize that the same argument holds for other banddirections. Also note that similar modulations in $C_{\phi}$ are present in any non-banded ordered state, as shown for $\eta_{\theta}=0.10$ in Fig. 4 a . However, we present our argument for the banded state only as band provides a precise measure of the relevant length and time scales of the flock dynamics. Therefore, the flock informations can be obtained from the modulations in $C_{\phi}$ of the NRs, where the amplitude and the frequency of the modulations are set by the ordering and the extent of the flock.

\section{Hydrodynamic description}

We develop a hydrodynamic framework for the nonquenched model described above. The relevant slow fields of the system with the NRs are - (i) density $\rho(\boldsymbol{r}, t)$ of the SPPs, (ii) polarisation $\boldsymbol{P}(\boldsymbol{r}, t)$ of the SPPs and (iii) polarisation $\boldsymbol{P}_{r}(\boldsymbol{r}, t)$ of the NRs. The density $\rho_{r}$ of the NRs is considered uniform as they are immobile and randomly distributed. Following the phenomenology of the system, we write the hydrodynamic equations of motion (EOMs) for the slow variables as follows:

$$
\begin{aligned}
\partial_{t} \rho & =-v_{s} \nabla \cdot(\rho \boldsymbol{P})+D_{\rho} \nabla^{2} \rho \\
\partial_{t} \boldsymbol{P}= & \left\{\alpha_{1}(\rho)-\beta_{1}\left|\boldsymbol{P}+\boldsymbol{P}_{r}\right|^{2}\right\} \boldsymbol{P}+\lambda_{1}(\boldsymbol{P} \cdot \nabla) \boldsymbol{P} \\
& \quad-\frac{v_{s}}{2 \rho} \nabla \rho+D \nabla^{2} \boldsymbol{P}+\gamma_{1} \rho \boldsymbol{P}_{r} \\
\partial_{t} \boldsymbol{P}_{r}= & \gamma_{2} \rho_{r} \boldsymbol{P}-\beta_{2}\left|\boldsymbol{P}+\boldsymbol{P}_{r}\right|^{2} \boldsymbol{P}_{r} .
\end{aligned}
$$

The density $\rho$ of the SPPs being a globally conserved quantity, Eq. (4) represents a continuity equation, however with an active current contribution 25. Here $v_{s}$ represents self-propulsion speed, and $D_{\rho}$ is the diffusion coefficient. As the ordering of the NRs affects the the SPPs, the mean-field term within the curly brackets in Eq. (5) contains both $\boldsymbol{P}$ and $\boldsymbol{P}_{r}$. We have considered only the $\lambda_{1}$ convective term as this is the most relevant convective nonlinearity [25, 37, 39]. The coefficient of the pressure term due to fluctuation in $\rho$ is taken $v_{s}$ for simplicity. Eq. (5) is written under equal-elastic-constant approximation [50]. The $\boldsymbol{P}_{r}$ term in Eq. (5) represents the feedback of the NRs to the SPPs that indeed depends on the density of the SPPs. Similar to this feedback term, the feedback of the SPPs to the NRs is represented by the first term on the right hand side of Eq. (6). The $\beta_{2}$ term in Eq. (6) stabilizes $\boldsymbol{P}_{r}$ in the steady state.

\section{Mean-field study}

Let us first consider a broken symmetry steady state such that a homogeneous solution of Eqs. (4)-(6) is given by $\rho=\bar{\rho}, \boldsymbol{P}=P \hat{\|}$, and $\boldsymbol{P}_{r}=P_{r} \hat{\|}$. Here $\|$ is the unit vector along the broken symmetry direction, and $\hat{\perp}$ is normal to that. For this homogeneous steady state, we obtain $P_{r}=B P$, where $B=\beta_{1} \gamma_{2} \rho_{r} / \alpha_{1}(\bar{\rho}) \beta_{2}$ (see Appendix B). Using this expression for $P_{r}$, we get

$$
P^{2}=\frac{\alpha_{1}(\bar{\rho})}{\beta_{1}} \times \frac{1+\gamma_{1} \bar{\rho} B / \alpha_{1}(\bar{\rho})}{(1+B)^{2}} .
$$

Note that $B=0$ for the clean system, and therefore the order parameter is $\mathcal{P}=\sqrt{\alpha_{1}(\bar{\rho}) / \beta_{1}}$. Therefore, the order parameter $P$ of the SPPs in the presence of the NRs is greater than its value $\mathcal{P}$ in the clean system, provided $\gamma_{1} \bar{\rho}-\beta_{1} \gamma_{2} \rho_{r} / \beta_{2}>2 \alpha_{1}(\bar{\rho})$, which indeed holds for an ordered state $\left(\alpha_{1}(\bar{\rho})>0\right)$. This necessarily implies a positive shift in the critical point, and therefore validates the existence of a more robust ordered state in the presence of the NRs.

\section{Linearised hydrodynamics}

Beyond the mean-field calculations, we consider small fluctuations in the slow fields:

$$
\begin{gathered}
\rho=\bar{\rho}+\delta \rho, \quad \boldsymbol{P}=\left(P+\delta P_{\|}, \delta P_{\perp}\right), \\
\boldsymbol{P}_{r}=\left(P_{r}+\delta P_{r \|}, \delta P_{r \perp}\right) .
\end{gathered}
$$

Simplifying the hydrodynamic EOMs under linearised approximation and solving for the fluctuations $\delta P_{\|}, \delta P_{r \|}$ and $\delta P_{r \perp}$, we obtain equations for $\delta \rho$ and $\delta P_{\perp}$ as

$$
\begin{gathered}
\partial_{t} \delta \rho=\left(D_{\|} \partial_{\|}^{2}+D_{\rho} \partial_{\perp}^{2}\right) \delta \rho-X v_{s} P \partial_{\|} \delta \rho-v_{s} \bar{\rho} \partial_{\perp} \delta P_{\perp} \\
\partial_{t} \delta P_{\perp}=D \nabla^{2} \delta P_{\perp}+\lambda_{1} P \partial_{\|} \delta P_{\perp}-\frac{v_{s}}{2 \bar{\rho}} \partial_{\perp} \delta \rho
\end{gathered}
$$

where

$$
D_{\|}=D_{\rho}+\frac{v_{s}^{2}}{2\left(\alpha_{1}^{\prime}-A^{\prime}\right)}, \quad X=1+\frac{\gamma_{1} B \bar{\rho}}{\alpha_{1}^{\prime}-A^{\prime}} .
$$

Here $D_{\|}$is an effective diffusivity, and the factor $X$ tunes the convective speed. In the clean system, $D_{\|}=$ 

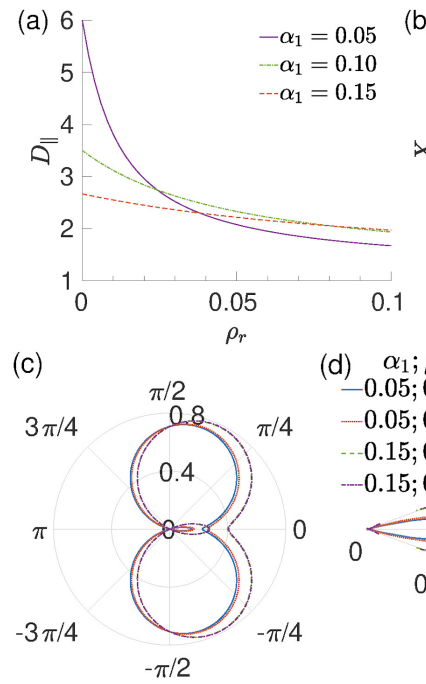

(d) $\begin{gathered}\alpha_{1} ; \rho_{r} \\ -0.05 ; 0.00\end{gathered}$

$-0.05 ; 0.10$

$-0.15 ; 0.00$

0

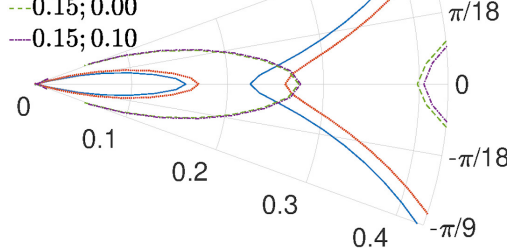

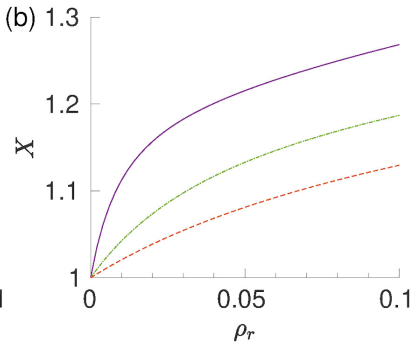

0.1

Figure 5. NRs increase sound-mode speeds. (a) The effective diffusivity $D_{\|}$decreases and (b) the factor $X$ increases with increasing density $\rho_{r}$ of heterogeneity. The changes are more dominant for smaller $\alpha_{1}(\bar{\rho})$, i.e., near the transition. Legends are the same in (a) and (b). (c) $c_{+}(\varphi)$ is plotted in polar coordinates for various $\alpha_{1}(\bar{\rho})$ and $\rho_{r}$, and its zoomed version is shown in $(\mathrm{d})$. The numerics adjacent to the radial and the angular grids indicate respective values, and help to realize the magnification in (d) as compared to (c). (c) and (d) share the same legends. The mode speed increases with $\rho_{r}$ along the flock direction, which is more prominent for small $\alpha_{1}(\bar{\rho})$.

$D_{\rho}+v_{s}^{2} / 4 \alpha_{1}(\bar{\rho})=\mathcal{D}$ and $X=1=\mathcal{X}$. For a finite $\rho_{r}$, $D_{\|}<\mathcal{D}$ and $X>\mathcal{X}$, as shown in Fig. 5 a-b (also see Appendix C). Therefore, the presence of the NRs reduces the effective diffusivity in the $\|$-direction and also increases the convective speed. These two modifications in the physical parameters are responsible for faster transfer of informations among the SPPs.

Solving Eqs. (9) and (10) in Fourier space 25, 37, we obtain two sound modes of the fluctuations as

$$
\omega_{ \pm}=c_{ \pm}(\varphi) q-i \Gamma_{\rho}\left[\frac{v_{ \pm}(\varphi)}{2 c_{2}(\varphi)}\right]-i \Gamma_{P}\left[\frac{v_{\mp}(\varphi)}{2 c_{2}(\varphi)}\right] .
$$

Here $\varphi$ is the angle between the wave vector $\boldsymbol{q}$ and the broken-symmetry direction so that $\boldsymbol{q}=(q \cos \varphi, q \sin \varphi)$, and

$$
\begin{aligned}
c_{2}(\varphi) & =\sqrt{\frac{1}{4}\left(X v_{s}+\lambda_{1}\right)^{2} P^{2} \cos ^{2} \varphi+\frac{1}{2} v_{s}^{2} \sin ^{2} \varphi}, \\
c_{ \pm}(\varphi) & =\frac{1}{2}\left(X v_{s}-\lambda_{1}\right) P \cos \varphi \pm c_{2}(\varphi), \\
v_{ \pm}(\varphi) & =c_{2}(\varphi) \pm \frac{1}{2}\left(X v_{s}-\lambda_{1}\right) P \cos \varphi .
\end{aligned}
$$

The wave-vector dependent dampings are $\Gamma_{\rho}(\boldsymbol{q})=$ $D_{\|} q_{\|}^{2}+D_{\rho} q_{\perp}$ and $\Gamma_{P}(\boldsymbol{q})=D q^{2}$. The sound speeds $c_{ \pm}(\varphi)$ differ mutually by a phase shift as $c_{+}(\varphi)=-c_{-}(\varphi+\pi)$. In Fig. 55-d we plot $c_{+}(\varphi)$ in polar coordinates for different values of $\alpha_{1}(\bar{\rho})$. For comparison we also plot $c_{+}$for

the clean system. Clearly, $c_{+}(\varphi)$ is larger for finite $\rho_{r}$, and the effect is the most dominant for $\varphi=0$. Also note that smaller the value of $\alpha_{1}(\bar{\rho})$, i.e., as we approach close to the critical point, more the change in sound speed. Therefore, the effect of the NRs are more prominent near the transition, as we have shown earlier in Fig. 2 a.

\section{Fluctuations in the NRs change the scenario}

We also study the effect of an additive noise in the $\phi$ update. Contrary to Eq. (3), the modified update rule for $\phi$ reads

$$
\phi_{j}(t+1)=\arg \left[e^{i \phi_{j}(t)}+\alpha \sum_{k \in R} e^{i \theta_{k}(t)}\right]+\eta_{\phi} \psi_{\phi} .
$$

The inclusion of this additional noise introduces randomness in the SPP-dynamics through the feedback and inertia mechanism discussed above. Consequently, the optimality feature analogous to the quenched model emerges in the system. The optimal $\eta_{\theta}$ for this modified model decreases linearly to zero as $\eta_{\phi}$ approaches zero. This verifies the claim of a monotonic order-disorder transition in the nonquenched model discussed previously in this article. We also note that $V_{s}$ decreases quadratically with $1 / N_{s}$ for this modified model, and an extrapolation of the quadratic fit suggests a finite $V_{s}$ in the thermodynamic limit. Therefore, we stress that the system remains in the LRO state for a finite $\eta_{\phi}$. We have discussed this phenomenology in details in Appendix D.

\section{DISCUSSION}

In summary, we study a polar self-propelled system with nonquenched rotators using a minimal rule-based microscopic model, and provide a hydrodynamic description of it. Although the self-propelled systems like a collection of bacteria or human crowds differ in their specific details [1 8, in general they follow common symmetries and conservation laws [15. Usually microscopic models 23, 44] are prolific in illustrating the common features of these systems, and hydrodynamic frameworks developed on the basis of the microscopic models make the descriptions more general.

In our model both the self-propelled particles (SPPs) and the rotators feedback each other their orientational informations. As the rotators memorise the passed-by subflock of SPPs and transfer that information to the next subflock, they offer an additional means of information transfer. Consequently, these rotators establish a long-range order flock more robust than the clean system. Note that in the absence of the inertia term in Eq. (3), a rotator 'forgets' the information of a passed-by flock immediately, and therefore, the reported robustness vanishes. The hydrodynamic framework of the nonquenched 
model verify the enhanced ordering using mean-field calculations. Further, considering linear fluctuations on a homogeneous ordered state, we show that the nonquenched heterogeneities decrease the effective diffusivity along flock-orientation that suppresses the fluctuations. Also, the heterogeneities increase the sound-mode speed which makes the transfer of information faster. These general results can be equally applicable to large social gatherings with similar nonquenched heterogeneities, and tuning the heterogeneities the panic or stampede like situations can be controlled.

Interestingly, the rotators store the information of the passed-by flock. Therefore, by probing a less number of heterogeneity agents, we can investigate the flockstatistics, as we have done by comparing the rotatorautocorrelation with the ordering and extent of the flock. This offers a novel mechanism for monitoring large crowd, alternative to bluetooth- or GPS-based methods [51 53].

\section{ACKNOWLEDGMENTS}

RD acknowledges CRAY supercomputing facility at S. N. Bose National Centre for Basic Sciences. SM acknowledges S. N. Bose National Centre for Basic Sciences for kind hospitality during her visit there.

\section{Appendix A: Determining $\eta_{\theta}^{c}$ from Binder cumulant}

We calculate Binder cumulant $U(L)$ for various system sizes. $U(L)$ assumes values $2 / 3$ and $1 / 3$, respectively, deep in the ordered and disordered states, as is expected for a 2D model with continuous rotational symmetry [26, 45. However, it dips to negative values near the transition, as shown in Fig. A.1, and it indicates the discontinuous nature of the transition. We assume the noise corresponding to the minimum of $U(L)$ as $\eta_{\theta}^{c}(L)$ which is expected to converge to the critical noise [26]. Note that $\eta_{\theta}^{c}(L)$ increases with $L$, which we fit using quadratic function as shown in Fig. 2 p.

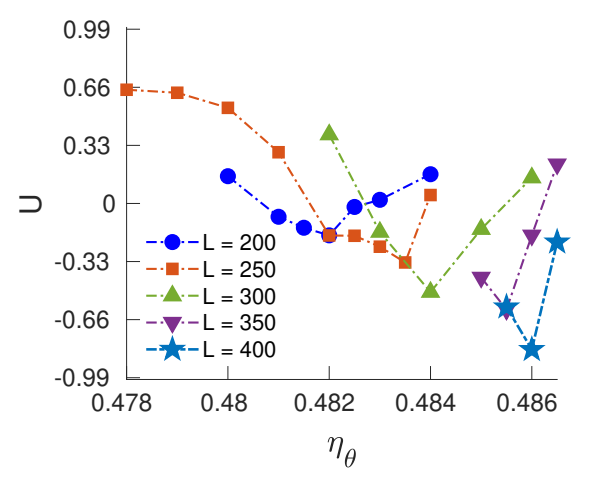

Figure A.1. Critical noise $\eta_{\theta}^{c}$ increases with $L$. Binder cumulants are shown for $c_{r}=0.008, \mu=200$ and $\alpha=1$, and zoomed near their minima for the sake of clarity.

\section{Appendix B: Mean-field hydrodynamics}

Considering a broken symmetry homogeneous steadystate solution of the hydrodynamic EOMs, as provided in the main text, and solving for the modulus $P$ and $P_{r}$ of the order parameter fields, we obtain

$$
\begin{aligned}
\alpha_{1}(\bar{\rho}) P+\gamma_{1} \bar{\rho} P_{r} & =\beta_{1}\left(P+P_{r}\right)^{2} P, \\
\left(P+P_{r}\right)^{2} & =\frac{\gamma_{2} \rho_{r} P}{\beta_{2} P_{r}} .
\end{aligned}
$$

Plugging the expression for $\left(P+P_{r}\right)^{2}$ from Eq. $\mathrm{B} 2$ into Eq. (B1), we obtain

$$
P_{r}=B P, \quad \text { where } B=\frac{\beta_{1} \gamma_{2}}{\alpha_{1}(\bar{\rho}) \beta_{2}} \rho_{r} .
$$

Using Eq. (B3), we obtain the expression for $P^{2}$, as in Eq. (7). This expression suggests enhance in the robustness of the system in the presence of the NRs.

\section{Appendix C: Linearised hydrodynamics}

We consider fluctuations in the slow fields, as written in the main text. Incorporating these fluctuations in the EOM for $\boldsymbol{P}_{r}$, and writing it for the $\|$ and $\perp$ components upto linear order in $B$, we obtain

$$
\delta P_{r \|}=A \delta P_{\|}, \quad \text { where } A=\frac{\gamma_{2} \rho_{r}-2 \beta_{2} B P^{2}}{\beta_{2}(1+4 B) P^{2}},
$$

and

$$
\delta P_{r \perp}=B \delta P_{\perp} .
$$

Similarly, writing the EOM for $\boldsymbol{P}$ for the $\|$-components and neglecting the higher order terms in fluctuations and derivatives, we get

$$
\delta P_{\|}=\frac{1}{\alpha_{1}^{\prime}-A^{\prime}}\left(\gamma_{1} B P-\frac{v_{s}}{2 \bar{\rho}} \partial_{\|}\right) \delta \rho,
$$

where

$$
\begin{aligned}
& \alpha_{1}^{\prime}=-\alpha_{1}(\bar{\rho})+\beta_{1}(1+B)^{2} P^{2}+2 \beta_{1}(1+B) P^{2}, \\
& A^{\prime}=\left\{\gamma_{1} \bar{\rho}-2 \beta_{1}(1+B) P^{2}\right\} A .
\end{aligned}
$$

Note that, $\alpha_{1}^{\prime}=2 \alpha_{1}(\bar{\rho})$ and $A^{\prime}$ vanishes for the clean system, and therefore, Eq. C3 takes the familiar form as in ref. 25.

Now as we have obtained the expressions for the fluctuations $\delta P_{\|}, \delta P_{r \|}$ and $\delta P_{r \perp}$, we solve for fluctuations $\delta P_{\perp}$ and $\delta \rho$. Plugging the above expressions into the $\rho$-equation (4), we obtain

$$
\partial_{t} \delta \rho=\left(D_{\|} \partial_{\|}^{2}+D_{\rho} \partial_{\perp}^{2}\right) \delta \rho-X v_{s} P \partial_{\|} \delta \rho-v_{s} \bar{\rho} \partial_{\perp} \delta P_{\perp}
$$


Also, writing the $\boldsymbol{P}$-equation (5) for the $\perp$-components upto linear order terms in fluctuations and simplifying it, we obtain

$$
\partial_{t} \delta P_{\perp}=D \nabla^{2} \delta P_{\perp}+\lambda_{1} P \partial_{\|} \delta P_{\perp}-\frac{v_{s}}{2 \bar{\rho}} \partial_{\perp} \delta \rho .
$$

Following the customary, we write Eqs. (C6) and (C7) in the Fourier space. Given a function $u(\boldsymbol{r}, t)$, its Fourier transform in space and time is defined as

$$
u(\boldsymbol{q}, \omega)=\int_{-\infty}^{\infty} d t d \boldsymbol{r} e^{i \omega t} e^{-i \boldsymbol{q} \cdot \boldsymbol{r}} u(\boldsymbol{r}, t) .
$$

Using this definition, we can write the EOMs for the fluctuations as

$$
M \Phi=\mathcal{O}
$$

where the fluctuation vector

$$
\boldsymbol{\Phi}=\left(\begin{array}{c}
\delta \rho(\boldsymbol{q}, \omega) \\
\delta P_{\perp}(\boldsymbol{q}, \omega)
\end{array}\right)
$$

and $\mathcal{O}$ represents the null vector. The coefficient matrix is given by

$$
\begin{aligned}
& \boldsymbol{M}(\boldsymbol{q}, \omega)= \\
& {\left[\begin{array}{cc}
i\left(\omega-X v_{s} P q_{\|}\right)-\Gamma_{\rho} & -i v_{\bar{\rho}} \bar{\rho} q_{\perp} \\
i v_{s} q_{\perp} / 2 \bar{\rho} & -i\left(\omega+\lambda_{1} P q_{\|}\right)+\Gamma_{P}
\end{array}\right] .}
\end{aligned}
$$

We obtain the normal modes of Eq. $(\mathrm{C} 9$ by solving $\operatorname{Det}[\boldsymbol{M}]=0$. This eventually gives a quadratic equation $\omega^{2}+b \omega+c=0$, where

$$
\begin{gathered}
b=\left(\lambda_{1}-X v_{s}\right) P q_{\|}+i\left(\Gamma_{P}+\Gamma_{\rho}\right), \\
c=-\lambda_{1} X v_{s} P^{2} q_{\|}^{2}-\frac{v_{s}^{2}}{2} q_{\perp}^{2}-\Gamma_{P} \Gamma_{\rho} \\
+i\left(\lambda_{1} \Gamma_{\rho}-X v_{s} \Gamma_{P}\right) P q_{\|} .
\end{gathered}
$$

The solution of this quadratic equation gives two sound modes $\omega_{ \pm}$with the convection speeds $c_{ \pm}$, as discussed in the main text.

\section{Appendix D: Fluctuations in the rotator update introduces randomness in the system}

Inclusion of the noise term $\eta_{\phi} \psi_{\phi}$ in the update Eq. (3) of NR-orientation induces the optimality feature, as the system attains the optimal ordering for a finite $\eta_{\theta}$ (Fig. D.1 1). The extra noise term in the $\phi$-update equation introduces randomness in the system that hinders mutual communications among the subflocks. Therefore, $V_{s}$ decreases with increasing $\eta_{\phi}$ (Fig. D.1 1 ). Provided the system has a finite $\eta_{\theta}$, the system overcomes the hindrance due to $\eta_{\phi}$ and attains the optimal order. The optimal noise $\eta_{\theta}^{\text {opt }}$ increases linearly with $\eta_{\phi}$ (Fig. D.1b). An extrapolation of the linear fit ensures the monotonic order-disorder transition for $\eta_{\phi}=0$.

Note that inclusion of the $\eta_{\phi}$-term makes $\phi$ behave like a colour noise to the SPP-orientation. As easily understood from the $\phi$-update rule, the autocorrelation of
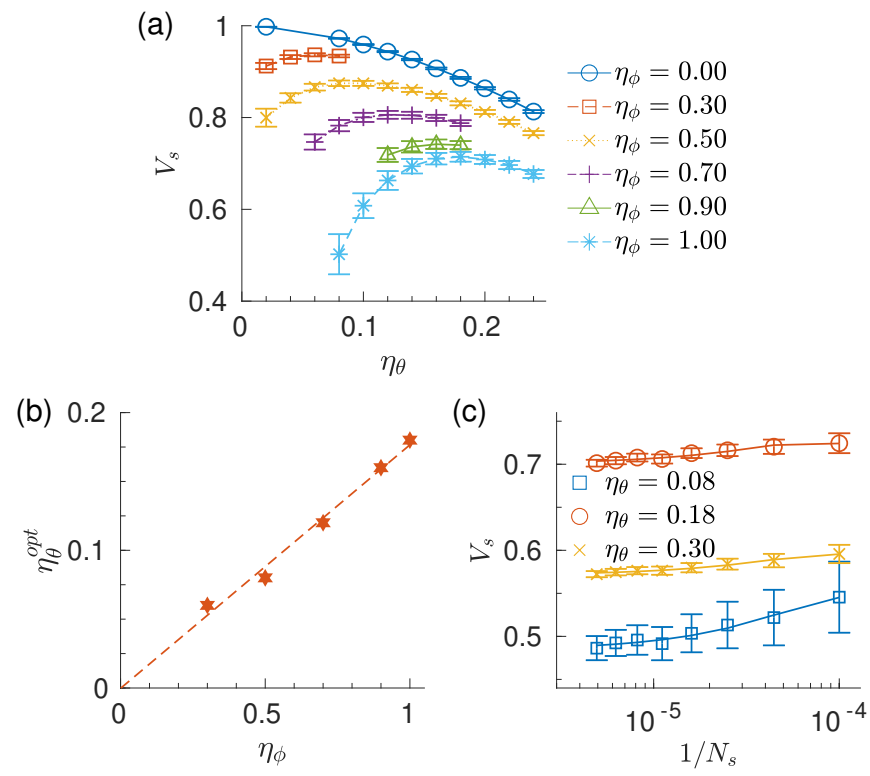

Figure D.1. $\quad \eta_{\phi}$-term introduces fluctuations in the system. (a) $V_{s}$ versus $\eta_{\theta}$ plot is shown for $c_{r}=0.01, \alpha=1$, and various $\eta_{\phi}$. The SPPs obtain optimal ordering at a finite $\eta_{\theta}$ in the presence of the $\eta_{\phi}$-term. The curves are obtained near the optimal point only, and the maximum $V_{s}$ suggests respective $\eta_{\theta}^{o p t}$. (b) $\eta_{\theta}^{o p t}$ varies linearly with $\eta_{\phi}$. The dashed line represents the extrapolation which verifies $\eta_{\theta}^{o p t}=0$ for $\eta_{\phi}=0$. (c) Variation in $V_{s}$ with system size is shown on semi-log scale for $c_{r}=0.01, \alpha=1$ and $\eta_{\phi}=1$. The solid lines show fits quadratic in $1 / N_{s}$ and have finite intercepts in the thermodynamic limit.

$\phi$ varies as $1 / \eta_{\phi}^{2}$ for $\alpha=0$. This sets in a timescale in the system that inhibits us to obtain a true steady state in a reasonable cpu-time. However, for a finite $\alpha$, the NRs suppress the effect of the colour noise and we obtain steady-states. We show the variation in $V_{s}$ with system size for $\alpha=1$ and the maximum noise $\eta_{\phi}=1$ in Fig. D.1. . We note that $V_{s}$ varies quadratically with $1 / N_{s}$, and the corresponding fits give finite $V_{s}$ as $1 / N_{s}=0$. Moreover, note that the fluctuation in $V_{s}$ decreases systematically with system size. Therefore, we argue that for finite $\alpha$ and finite $\eta_{\phi}$, the system will obtain a long-range order state; however, the presence of the $\eta_{\phi}$-term inhibits us to find it unambiguously.

\section{REFERENCES}

[1] I. Barnett, T. Khanna and J.-P. Onnela, Social and Spatial Clustering of People at Humanity's Largest Gathering, PLoS One 11, e0156794 (2016).

[2] R. L. Hughes, The Flow of Human Crowds, Annu. Rev. Fluid Mech. 35, 169 (2003).

[3] S. Kim, S. J. Guy, K. Hillesland, B. Zafar, A. A.-A. Gutub and D. Manocha, Velocity-based modeling of physical interactions in dense crowds, Visual Computer 31, 541 (2015).

[4] S. Curtis, S. J. Guy, B. Zafar and D. Manocha, in Model- 
ing, Simulation and Visual Analysis of Crowds: A Multidisciplinary Perspective, edited by S. Ali, K. Nishino, D.Manocha and M. Shah (Springer, New York, 2013), pp. 181209.

[5] A. Baranwal et al., Managing the Earths Biggest Mass Gathering Event and WASH Conditions: Maha Kumbh Mela (India), PLoS Currents Disasters 7 (2015).

[6] A. Cavagna and I. Giardina, Bird Flocks as Condensed Matter, Annu. Rev. Condens. Matter Phys. 5, 183 (2014).

[7] C. Dombrowski, L. Cisneros, S. Chatkaew, R. E. Goldstein and J. O. Kessler, Self-Concentration and LargeScale Coherence in Bacterial Dynamics, Phys. Rev. Lett. 93, 098103 (2004).

[8] D. Asem et al., Evaluation of gastrointestinal bacterial population for the production of holocellulose enzymes for biomass deconstruction, PLoS One 12, e0186355 (2017).

[9] J. U. Klamser, S. C. Kapfer and W. Krauth, Thermodynamic phases in two-dimensional active matter, Nat. Comm. 9, 5045 (2018).

[10] M. E. Cates and J. Tailleur, Motility-Induced Phase Separation, Annu. Rev. Condens. Matter Phys. 6, 219 (2015).

[11] F. Bergmann, L. Rapp and W. Zimmermann, Active phase separation: A universal approach, Phys. Rev. E 98, 020603(R) (2018).

[12] S. Dey, D. Das and R. Rajesh, Spatial Structures and Giant Number Fluctuations in Models of Active Matter, Phys. Rev. Lett. 108, 238001 (2012).

[13] M. C. Marchetti et al., Hydrodynamics of soft active matter, Rev. Mod. Phys. 85, 1143 (2013).

[14] J. Toner, Y. Tu and S. Ramaswamy, Hydrodynamics and phases of flocks, Ann. Phys. 318, 170 (2005).

[15] S. Ramaswamy, The Mechanics and Statistics of Active Matter, Annu. Rev. Cond. Matt. Phys. 1, 323 (2010).

[16] T. Vicsek and A. Zafeiris, Collective motion, Phys. Rep. 517, 71 (2012).

[17] M. E. Cates, Diffusive transport without detailed balance in motile bacteria: does microbiology need statistical physics?, Rep. Prog. Phys. 75, 042601 (2012).

[18] G. Popkin, The physics of life,Nature 529, 16 (2016).

[19] D. Needleman and Z. Dogic, Active matter at the interface between materials science and cell biology, Nat. Rev. Mat. 2, 17048 (2017).

[20] W. Xi, T. B. Saw, D. Delacour, C. T. Lim and B. Ladoux, Material approaches to active tissue mechanics, Nat. Rev. Mat. 4, 23 (2019).

[21] H. Lu et al., A bioinspired multilegged soft millirobot that functions in both dry and wet conditions, Nat. Comm. 9, 3944 (2018).

[22] R. Singh and J. W. Jr. Lillard, Nanoparticle-based targeted drug delivery, Exp. Mol. Pathol. 86, 215 (2009).

[23] T. Vicsek, A. Czirók, E. Ben-Jacob, I. Cohen and O. Shochet, Novel Type of Phase Transition in a System of Self-Driven Particles, Phys. Rev. Lett. 75, 1226 (1995).

[24] J. Toner and Y. Tu, Long-Range Order in a TwoDimensional Dynamical XY Model: How Birds Fly Together, Phys. Rev. Lett. 75, 4326 (1995).

[25] J. Toner and Y. Tu, Flocks, herds, and schools: A quantitative theory of flocking, Phys. Rev. E 58, 4828 (1998).

[26] H. Chaté, F. Ginelli, G. Grégoire and F. Raynaud, Collective motion of self-propelled particles interacting without cohesion, Phys. Rev. E 77, 046113 (2008).

[27] V. Schaller, C. Weber, C. Semmrich, E. Frey and A. R. Bausch, Polar patterns of driven filaments, Nature 467, 73 (2010).
[28] C. J. O. Reichhardt and C. Reichhardt, Disorder in the wild, Nat. Phys. 13, 10 (2017).

[29] J. C. Stearns et al., Bacterial biogeography of the human digestive tract, Sci. Rep. 1, 170 (2011).

[30] W. Pönisch, C. A. Weber and V. Zaburdaev, How bacterial cells and colonies move on solid substrates, Phys. Rev. E 99, 042419 (2019).

[31] A. Garcimartín et al., Redefining the role of obstacles in pedestrian evacuation, New J. Phys 20, 123025 (2018).

[32] L. Jiang, J. Li, C. Shen, S. Yang and Z. Han, Obstacle Optimization for Panic Flow - Reducing the Tangential Momentum Increases the Escape Speed, PLoS One 9, e115463 (2014).

[33] A. Morin, N. Desreumaux, J. B. Caussin and D. Bartolo, Distortion and destruction of colloidal flocks in disordered environments, Nat. Phys. 13, 63 (2017).

[34] O. Chepizhko, E. G. Altmann and F. Peruani, Optimal Noise Maximizes Collective Motion in Heterogeneous Media, Phys. Rev. Lett. 110, 238101 (2013).

[35] D. Yllanes, M. Leoni and M. C. Marchetti, How many dissenters does it take to disorder a flock? New J. Phys. 19, 103026 (2017).

[36] D. A. Quint and A. Gopinathan, Topologically induced swarming phase transition on a 2D percolated lattice, Phys. Biol. 12, 046008 (2015).

[37] R. Das, M. Kumar and S. Mishra, Polar flock in the presence of random quenched rotators, Phys. Rev. E 98, 060602(R) (2018).

[38] J. Toner, N. Guttenberg and Y. Tu, Swarming in the Dirt: Ordered Flocks with Quenched Disorder, Phys. Rev. Lett. 121, 248002 (2018).

[39] J. Toner, N. Guttenberg and Y. Tu, Hydrodynamic theory of flocking in the presence of quenched disorder, Phys. Rev. E 98, 062604 (2018).

[40] A. Kulkarni, S. P. Thampi and M. V. Panchagnula, Sparse Game Changers Restore Collective Motion in Panicked Human Crowds, Phys. Rev. Lett. 122, 048002 (2019).

[41] R. Yano, Effect of form of obstacle on speed of crowd evacuation, Phys. Rev. E 97, 032319 (2018).

[42] I. Zuriguel et al., Silo Clogging Reduction by the Presence of an Obstacle, Phys. Rev. Lett. 107, 178001 (2011).

[43] P. Lin et al., An experimental study of the impact of an obstacle on the escape efficiency by using mice under high competition, Physica A 482, 228 (2017).

[44] G. Grégoire and H. Chaté, Onset of Collective and Cohesive Motion, Phys. Rev. Lett. 92, 025702 (2004).

[45] M. Aldana, H. Larralde and B. Vázquez, On the Emergence of Collective Order in Swarming Systems: A Recent Debate, Int. Journ. Mod. Phys. B 23, 3661 (2009).

[46] K. Binder, Critical Properties from Monte Carlo Coarse Graining and Renormalization, Phys. Rev. Lett. 47, 693 (1981).

[47] A. P. Solon, H. Chaté and J. Tailleur, From Phase to Microphase Separation in Flocking Models: The Essential Role of Nonequilibrium Fluctuations, Phys. Rev. Lett. 114, 068101 (2015).

[48] A. P. Solon and J. Tailleur, Flocking with discrete symmetry: The two-dimensional active Ising model, Phys. Rev. E 92, 042119 (2015).

[49] The top panel in the Supplementary Movie shows modulations in $C_{\phi}$ of the NRs for a banded state. The snapshot corresponding to the red dot over the modulations is shown in the bottom panel. The snapshot is shown for a 
$300 \times 300$ substrate and only a few NRs are drawn for the sake of clarity. The red arrow on the top margin of the snapshot-panel indicates the position of the band-front at the starting time of the movie. The colourbar indicates orientations of the SPPs and the NRs. Note that the NRs inside the band have similar (correlated) orientations due the feedback from the ordered SPPs. However, outside the band, the NRs have random orientations as they are interacting with disordered fluid of SPPs. $C_{\phi}$ has finite values only when the current band-position overlaps with the position at the starting point.

[50] P. G. de Gennes and J. Prost, in The Physics of Liquid
Crystals (Oxford University Press, New York, 1993).

[51] S. K. Shah and S. Kulkarni, A Review: Monitoring and Safety of Pilgrims Using Stampede Detection and Pilgrim Tracking, International Journal of Research in Engineering and Technology 4, 328 (2015).

[52] G. Dell'Ariccia, G, Dell'Omo, D. P. Wolfer and H.-P. Lipp, Flock flying improves pigeons' homing: GPS track analysis of individual flyers versus small groups, Animal Behaviour 76, 1165 (2008).

[53] A. Flack, M. Nagy, W. Fiedler, I. D. Couzin and M. Wikelski, From local collective behavior to global migratory patterns in white storks, Science 360, 911 (2018). 